\title{
Simultaneous Effect of Microemulsions and Phase Transfer Agents on Aminolysis Reactions
}

L. García-Río, J. C. Mejuto, M. Pérez-Lorenzo

\begin{tabular}{|l|r|}
\hline $\begin{array}{l}\text { Influence of }\left[\mathrm{BuNH}_{2}\right] \text { and }[\mathrm{Glyme}] \text { on } k_{\text {obs }} \text { for butylaminolysis of NPC } \\
\text { in chlorobenzene }\end{array}$ & $\mathrm{S} 2$ \\
\hline $\begin{array}{l}\text { Influence of }\left[\mathrm{BuNH}_{2}\right] \text { and }[\mathrm{Glyme}] \text { on } k_{\text {obs }} \text { for butylaminolysis of NPC } \\
\text { in AOT/chlorobenzene/water microemulsions. } W=2 .\end{array}$ & $\mathrm{S} 4$ \\
\hline $\begin{array}{l}\text { Influence of }\left[\mathrm{BuNH}_{2}\right] \text { and }[\mathrm{Glyme}] \text { on } k_{\text {obs }} \text { for butylaminolysis of NPC } \\
\text { in AOT/chlorobenzene/water microemulsions. } W=6 .\end{array}$ & $\mathrm{S} 14$ \\
\hline Derivation of the kinetic equation in the absence of phase transfer catalyst & $\mathrm{S} 22$ \\
\hline Derivation of the kinetic equation in the presence of phase transfer catalyst & $\mathrm{S} 28$ \\
\hline
\end{tabular}


Table S-1. Influence of $n$-butylamine concentration of $k_{o b s}$ for butylaminolysis of NPC in chlorobenzene in the presence of different glyme concentrations. $[\mathrm{NPC}]=5.0 \times 10^{-5} \mathrm{M}$. $\mathrm{T}=25.0^{\circ} \mathrm{C}$.

\begin{tabular}{|c|c|c|}
\hline$[\mathbf{G}], \mathbf{M}$ & {$\left[\mathrm{BuNH}_{2}\right], \mathbf{M}$} & $k_{o b s}, \mathbf{s}^{-1}$ \\
\hline 0.00 & 0.02 & $7.701 \times 10^{-6}$ \\
\hline 0.00 & 0.06 & $6.668 \times 10^{-5}$ \\
\hline 0.00 & 0.10 & $1.894 \times 10^{-4}$ \\
\hline 0.00 & 0.15 & $4.188 \times 10^{-4}$ \\
\hline 0.00 & 0.22 & $9.657 \times 10^{-4}$ \\
\hline 0.00 & 0.30 & $1.948 \times 10^{-3}$ \\
\hline 0.00 & 0.40 & $3.413 \times 10^{-3}$ \\
\hline 0.10 & 0.02 & $1.302 \times 10^{-4}$ \\
\hline 0.10 & 0.06 & $5.185 \times 10^{-4}$ \\
\hline 0.10 & 0.10 & $9.191 \times 10^{-4}$ \\
\hline 0.10 & 0.15 & $1.603 \times 10^{-3}$ \\
\hline 0.10 & 0.22 & $2.768 \times 10^{-3}$ \\
\hline 0.10 & 0.30 & $4.376 \times 10^{-3}$ \\
\hline 0.10 & 0.40 & $7.369 \times 10^{-3}$ \\
\hline 0.25 & 0.02 & $3.559 \times 10^{-4}$ \\
\hline 0.25 & 0.06 & $1.376 \times 10^{-3}$ \\
\hline 0.25 & 0.10 & $2.280 \times 10^{-3}$ \\
\hline 0.25 & 0.15 & $3.702 \times 10^{-3}$ \\
\hline 0.25 & 0.22 & $5.873 \times 10^{-3}$ \\
\hline 0.25 & 0.30 & $8.879 \times 10^{-3}$ \\
\hline 0.25 & 0.40 & $1.346 \times \cdot 10^{-2}$ \\
\hline
\end{tabular}


Table S-1. Continuation.

\begin{tabular}{|c|c|c|}
\hline$[\mathbf{G}], \mathbf{M}$ & {$\left[\mathrm{BuNH}_{2}\right], \mathbf{M}$} & $k_{o b s}, \mathbf{s}^{-1}$ \\
\hline 0.50 & 0.02 & $7.392 \times 10^{-4}$ \\
\hline 0.50 & 0.06 & $2.665 \times 10^{-3}$ \\
\hline 0.50 & 0.10 & $4.627 \times 10^{-3}$ \\
\hline 0.50 & 0.15 & $7.340 \times 10^{-3}$ \\
\hline 0.50 & 0.22 & $1.156 \times 10^{-2}$ \\
\hline 0.50 & 0.30 & $1.696 \times 10^{-2}$ \\
\hline 0.50 & 0.40 & $2.457 \times 10^{-2}$ \\
\hline 0.75 & 0.02 & $1.269 \times 10^{-3}$ \\
\hline 0.75 & 0.06 & $4.017 \times 10^{-3}$ \\
\hline 0.75 & 0.10 & $7.017 \times 10^{-3}$ \\
\hline 0.75 & 0.15 & $1.102 \times 10^{-2}$ \\
\hline 0.75 & 0.22 & $1.701 \times 10^{-2}$ \\
\hline 0.75 & 0.30 & $2.465 \times 10^{-2}$ \\
\hline 0.75 & 0.40 & $3.545 \times 10^{-2}$ \\
\hline 1.00 & 0.02 & $1.689 \times 10^{-3}$ \\
\hline 1.00 & 0.06 & $5.753 \times 10^{-3}$ \\
\hline 1.00 & 0.10 & $9.937 \times 10^{-3}$ \\
\hline 1.00 & 0.15 & $1.532 \times 10^{-2}$ \\
\hline 1.00 & 0.22 & $2.356 \times 10^{-2}$ \\
\hline 1.00 & 0.30 & $3.266 \times 10^{-2}$ \\
\hline 1.00 & 0.40 & $4.537 \times 10^{-2}$ \\
\hline
\end{tabular}


Table S-2. Influence of $n$-butylamine concentration of $k_{o b s}$ for butylaminolysis of NPC in $\mathrm{AOT} /$ chlorobenzene/water microemulsions in the presence of different glyme concentration. $[\mathrm{NPC}]=5.0 \times 10^{-5} \mathrm{M} . \mathrm{T}=25.0^{\circ} \mathrm{C} . W=2$.

\begin{tabular}{|c|c|c|c|c|c|}
\hline$W$ & {$[\mathrm{AOT}], \mathbf{M}$} & $Z$ & {$[\mathbf{G}], \mathbf{M}$} & {$\left[\mathrm{BuNH}_{2}\right], \mathbf{M}$} & $k_{o b s}, \mathbf{s}^{-1}$ \\
\hline 2 & 0.10 & 92.957 & 0.00 & 0.10 & $3.317 \times 10^{-4}$ \\
\hline 2 & 0.10 & 92.469 & 0.00 & 0.15 & $6.563 \times 10^{-4}$ \\
\hline 2 & 0.10 & 91.005 & 0.00 & 0.30 & $2.583 \times 10^{-3}$ \\
\hline 2 & 0.10 & 90.029 & 0.00 & 0.40 & $4.628 \times 10^{-3}$ \\
\hline 2 & 0.10 & 91.163 & 0.10 & 0.10 & $1.319 \times 10^{-3}$ \\
\hline 2 & 0.10 & 90.675 & 0.10 & 0.15 & $2.225 \times 10^{-3}$ \\
\hline 2 & 0.10 & 89.211 & 0.10 & 0.30 & $5.973 \times 10^{-3}$ \\
\hline 2 & 0.10 & 88.235 & 0.10 & 0.40 & $9.171 \times 10^{-3}$ \\
\hline 2 & 0.10 & 88.472 & 0.25 & 0.10 & $3.104 \times 10^{-3}$ \\
\hline 2 & 0.10 & 87.984 & 0.25 & 0.15 & $4.792 \times 10^{-3}$ \\
\hline 2 & 0.10 & 86.521 & 0.25 & 0.30 & $1.159 \times 10^{-2}$ \\
\hline 2 & 0.10 & 85.545 & 0.25 & 0.40 & $1.632 \times 10^{-2}$ \\
\hline 2 & 0.10 & 83.988 & 0.50 & 0.10 & $5.792 \times 10^{-3}$ \\
\hline 2 & 0.10 & 83.500 & 0.50 & 0.15 & $9.284 \times 10^{-3}$ \\
\hline 2 & 0.10 & 82.036 & 0.50 & 0.30 & $2.082 \times 10^{-2}$ \\
\hline 2 & 0.10 & 81.060 & 0.50 & 0.40 & $2.916 \times 10^{-2}$ \\
\hline 2 & 0.10 & 79.504 & 0.75 & 0.10 & $9.610 \times 10^{-3}$ \\
\hline 2 & 0.10 & 79.016 & 0.75 & 0.15 & $1.447 \times 10^{-2}$ \\
\hline 2 & 0.10 & 77.552 & 0.75 & 0.30 & $3.208 \times 10^{-2}$ \\
\hline 2 & 0.10 & 76.576 & 0.75 & 0.40 & $4.327 \times 10^{-2}$ \\
\hline
\end{tabular}


Table S-2. Continuation.

\begin{tabular}{|c|c|c|c|c|c|}
\hline $\boldsymbol{W}$ & {$[\mathrm{AOT}], \mathrm{M}$} & $Z$ & {$[\mathbf{G}], \mathbf{M}$} & {$\left[\mathrm{BuNH}_{2}\right], \mathbf{M}$} & $k_{o b s}, s^{-1}$ \\
\hline 2 & 0.10 & 75.020 & 1.00 & 0.10 & $1.213 \times 10^{-2}$ \\
\hline 2 & 0.10 & 74.532 & 1.00 & 0.15 & $1.824 \times 10^{-2}$ \\
\hline 2 & 0.10 & 73.068 & 1.00 & 0.30 & $4.007 \times 10^{-2}$ \\
\hline 2 & 0.10 & 72.092 & 1.00 & 0.40 & $5.638 \times 10^{-2}$ \\
\hline 2 & 0.15 & 60.901 & 0.00 & 0.06 & $1.811 \times 10^{-4}$ \\
\hline 2 & 0.15 & 60.641 & 0.00 & 0.10 & $3.585 \times 10^{-4}$ \\
\hline 2 & 0.15 & 60.316 & 0.00 & 0.15 & $7.031 \times 10^{-4}$ \\
\hline 2 & 0.15 & 59.860 & 0.00 & 0.22 & $1.435 \times 10^{-3}$ \\
\hline 2 & 0.15 & 59.340 & 0.00 & 0.30 & $2.562 \times 10^{-3}$ \\
\hline 2 & 0.15 & 58.690 & 0.00 & 0.40 & $4.609 \times 10^{-3}$ \\
\hline 2 & 0.15 & 59.705 & 0.10 & 0.06 & $6.819 \times 10^{-4}$ \\
\hline 2 & 0.15 & 59.446 & 0.10 & 0.10 & $1.262 \times 10^{-3}$ \\
\hline 2 & 0.15 & 59.120 & 0.10 & 0.15 & $2.015 \times 10^{-3}$ \\
\hline 2 & 0.15 & 58.664 & 0.10 & 0.22 & $3.578 \times 10^{-3}$ \\
\hline 2 & 0.15 & 58.144 & 0.10 & 0.30 & $5.762 \times 10^{-3}$ \\
\hline 2 & 0.15 & 57.494 & 0.10 & 0.40 & $8.457 \times 10^{-3}$ \\
\hline 2 & 0.15 & 57.912 & 0.25 & 0.06 & $1.609 \times 10^{-3}$ \\
\hline 2 & 0.15 & 57.652 & 0.25 & 0.10 & $2.773 \times 10^{-3}$ \\
\hline 2 & 0.15 & 57.327 & 0.25 & 0.15 & $4.285 \times 10^{-3}$ \\
\hline 2 & 0.15 & 56.870 & 0.25 & 0.22 & $6.880 \times 10^{-3}$ \\
\hline 2 & 0.15 & 56.351 & 0.25 & 0.30 & $1.035 \times 10^{-2}$ \\
\hline 2 & 0.15 & 55.700 & 0.25 & 0.40 & $1.570 \times 10^{-2}$ \\
\hline
\end{tabular}


Table S-2. Continuation.

\begin{tabular}{|c|c|c|c|c|c|}
\hline $\boldsymbol{W}$ & {$[\mathrm{AOT}], \mathrm{M}$} & $Z$ & {$[\mathbf{G}], \mathbf{M}$} & {$\left[\mathrm{BuNH}_{2}\right], \mathbf{M}$} & $k_{o b s}, s^{-1}$ \\
\hline 2 & 0.15 & 54.922 & 0.50 & 0.06 & $3.312 \times 10^{-3}$ \\
\hline 2 & 0.15 & 54.662 & 0.50 & 0.10 & $5.774 \times 10^{-3}$ \\
\hline 2 & 0.15 & 54.337 & 0.50 & 0.15 & $8.851 \times 10^{-3}$ \\
\hline 2 & 0.15 & 53.881 & 0.50 & 0.22 & $1.381 \times 10^{-2}$ \\
\hline 2 & 0.15 & 53.361 & 0.50 & 0.30 & $1.987 \times 10^{-2}$ \\
\hline 2 & 0.15 & 52.711 & 0.50 & 0.40 & $2.847 \times 10^{-2}$ \\
\hline 2 & 0.15 & 51.933 & 0.75 & 0.06 & $5.073 \times 10^{-3}$ \\
\hline 2 & 0.15 & 51.673 & 0.75 & 0.10 & $8.903 \times 10^{-3}$ \\
\hline 2 & 0.15 & 51.348 & 0.75 & 0.15 & $1.336 \times 10^{-2}$ \\
\hline 2 & 0.15 & 50.891 & 0.75 & 0.22 & $2.099 \times 10^{-2}$ \\
\hline 2 & 0.15 & 50.372 & 0.75 & 0.30 & $3.123 \times 10^{-2}$ \\
\hline 2 & 0.15 & 49.721 & 0.75 & 0.40 & $4.007 \times 10^{-2}$ \\
\hline 2 & 0.15 & 48.943 & 1.00 & 0.06 & $7.499 \times 10^{-3}$ \\
\hline 2 & 0.15 & 48.683 & 1.00 & 0.10 & $1.223 \times 10^{-2}$ \\
\hline 2 & 0.15 & 48.358 & 1.00 & 0.15 & $1.849 \times 10^{-2}$ \\
\hline 2 & 0.15 & 47.902 & 1.00 & 0.22 & $2.910 \times 10^{-2}$ \\
\hline 2 & 0.15 & 47.382 & 1.00 & 0.30 & $4.028 \times 10^{-2}$ \\
\hline 2 & 0.15 & 46.732 & 1.00 & 0.40 & $5.897 \times 10^{-2}$ \\
\hline 2 & 0.20 & 44.874 & 0.00 & 0.02 & $5.504 \times 10^{-5}$ \\
\hline 2 & 0.20 & 44.679 & 0.00 & 0.06 & $2.078 \times 10^{-4}$ \\
\hline 2 & 0.20 & 44.484 & 0.00 & 0.10 & $4.208 \times 10^{-4}$ \\
\hline 2 & 0.20 & 44.240 & 0.00 & 0.15 & $7.760 \times 10^{-4}$ \\
\hline
\end{tabular}


Table S-2. Continuation.

\begin{tabular}{|c|c|c|c|c|c|}
\hline$W$ & {$[\mathbf{A O T}], \mathbf{M}$} & $Z$ & {$[\mathbf{G}], \mathbf{M}$} & {$\left[\mathrm{BuNH}_{2}\right], \mathbf{M}$} & $k_{o b s}, \mathbf{s}^{-1}$ \\
\hline 2 & 0.20 & 43.898 & 0.00 & 0.22 & $1.455 \times 10^{-3}$ \\
\hline 2 & 0.20 & 43.508 & 0.00 & 0.30 & $2.712 \times 10^{-3}$ \\
\hline 2 & 0.20 & 43.020 & 0.00 & 0.40 & $4.759 \times 10^{-3}$ \\
\hline 2 & 0.20 & 43.977 & 0.10 & 0.02 & $2.185 \times 10^{-4}$ \\
\hline 2 & 0.20 & 43.782 & 0.10 & 0.06 & $7.015 \times 10^{-4}$ \\
\hline 2 & 0.20 & 43.587 & 0.10 & 0.10 & $1.247 \times 10^{-3}$ \\
\hline 2 & 0.20 & 43.343 & 0.10 & 0.15 & $2.044 \times 10^{-3}$ \\
\hline 2 & 0.20 & 43.001 & 0.10 & 0.22 & $3.730 \times 10^{-3}$ \\
\hline 2 & 0.20 & 42.611 & 0.10 & 0.30 & $5.547 \times 10^{-3}$ \\
\hline 2 & 0.20 & 42.123 & 0.10 & 0.40 & $8.608 \times 10^{-3}$ \\
\hline 2 & 0.20 & 42.632 & 0.25 & 0.02 & $4.534 \times 10^{-4}$ \\
\hline 2 & 0.20 & 42.437 & 0.25 & 0.06 & $1.627 \times 10^{-3}$ \\
\hline 2 & 0.20 & 42.242 & 0.25 & 0.10 & $2.801 \times 10^{-3}$ \\
\hline 2 & 0.20 & 41.998 & 0.25 & 0.15 & $4.453 \times 10^{-3}$ \\
\hline 2 & 0.20 & 41.656 & 0.25 & 0.22 & $7.221 \times 10^{-3}$ \\
\hline 2 & 0.20 & 41.266 & 0.25 & 0.30 & $1.081 \times 10^{-2}$ \\
\hline 2 & 0.20 & 40.778 & 0.25 & 0.40 & $1.570 \times 10^{-2}$ \\
\hline 2 & 0.20 & 40.390 & 0.50 & 0.02 & $1.013 \times 10^{-3}$ \\
\hline 2 & 0.20 & 40.194 & 0.50 & 0.06 & $3.280 \times 10^{-3}$ \\
\hline 2 & 0.20 & 40.000 & 0.50 & 0.10 & $5.842 \times 10^{-3}$ \\
\hline 2 & 0.20 & 39.756 & 0.50 & 0.15 & $9.234 \times 10^{-3}$ \\
\hline 2 & 0.20 & 39.413 & 0.50 & 0.22 & $1.430 \times 10^{-2}$ \\
\hline 2 & 0.20 & 39.024 & 0.50 & 0.30 & $2.124 \times 10^{-2}$ \\
\hline 2 & 0.20 & 38.536 & 0.50 & 0.40 & $2.974 \times 10^{-2}$ \\
\hline
\end{tabular}


Table S-2. Continuation.

\begin{tabular}{|c|c|c|c|c|c|}
\hline$W$ & {$[\mathbf{A O T}], \mathbf{M}$} & $Z$ & {$[\mathbf{G}], \mathbf{M}$} & {$\left[\mathrm{BuNH}_{2}\right], \mathbf{M}$} & $k_{o b s}, \mathbf{s}^{-1}$ \\
\hline 2 & 0.20 & 38.148 & 0.75 & 0.02 & $1.800 \times 10^{-3}$ \\
\hline 2 & 0.20 & 37.952 & 0.75 & 0.06 & $5.585 \times 10^{-3}$ \\
\hline 2 & 0.20 & 37.757 & 0.75 & 0.10 & $9.591 \times 10^{-3}$ \\
\hline 2 & 0.20 & 37.514 & 0.75 & 0.15 & $1.466 \times 10^{-2}$ \\
\hline 2 & 0.20 & 37.171 & 0.75 & 0.22 & $2.262 \times 10^{-2}$ \\
\hline 2 & 0.20 & 36.782 & 0.75 & 0.30 & $3.342 \times 10^{-2}$ \\
\hline 2 & 0.20 & 36.294 & 0.75 & 0.40 & $4.502 \times 10^{-2}$ \\
\hline 2 & 0.20 & 35.906 & 1.00 & 0.02 & $2.634 \times 10^{-3}$ \\
\hline 2 & 0.20 & 35.710 & 1.00 & 0.06 & $8.045 \times 10^{-3}$ \\
\hline 2 & 0.20 & 35.515 & 1.00 & 0.10 & $1.370 \times 10^{-2}$ \\
\hline 2 & 0.20 & 35.271 & 1.00 & 0.15 & $2.088 \times 10^{-2}$ \\
\hline 2 & 0.20 & 34.929 & 1.00 & 0.22 & $3.240 \times 10^{-2}$ \\
\hline 2 & 0.20 & 34.539 & 1.00 & 0.30 & $4.573 \times 10^{-2}$ \\
\hline 2 & 0.20 & 34.051 & 1.00 & 0.40 & $6.352 \times 10^{-2}$ \\
\hline 2 & 0.30 & 28.587 & 0.00 & 0.02 & $6.787 \times 10^{-5}$ \\
\hline 2 & 0.30 & 28.456 & 0.00 & 0.06 & $2.351 \times 10^{-4}$ \\
\hline 2 & 0.30 & 28.326 & 0.00 & 0.10 & $4.665 \times 10^{-4}$ \\
\hline 2 & 0.30 & 28.164 & 0.00 & 0.15 & $8.543 \times 10^{-4}$ \\
\hline 2 & 0.30 & 27.935 & 0.00 & 0.22 & $1.654 \times 10^{-3}$ \\
\hline 2 & 0.30 & 27.676 & 0.00 & 0.30 & $2.830 \times 10^{-3}$ \\
\hline 2 & 0.30 & 27.350 & 0.00 & 0.40 & $4.939 \times 10^{-3}$ \\
\hline
\end{tabular}


Table S-2. Continuation.

\begin{tabular}{|c|c|c|c|c|c|}
\hline $\boldsymbol{W}$ & {$[\mathrm{AOT}], \mathbf{M}$} & $Z$ & {$[\mathbf{G}], \mathbf{M}$} & {$\left[\mathrm{BuNH}_{2}\right], \mathbf{M}$} & $k_{o b s}, \mathrm{~s}^{-1}$ \\
\hline 2 & 0.30 & 27.989 & 0.10 & 0.02 & $2.267 \times 10^{-4}$ \\
\hline 2 & 0.30 & 27.858 & 0.10 & 0.06 & $7.177 \times 10^{-4}$ \\
\hline 2 & 0.30 & 27.728 & 0.10 & 0.10 & $1.304 \times 10^{-3}$ \\
\hline 2 & 0.30 & 27.566 & 0.10 & 0.15 & $2.146 \times 10^{-3}$ \\
\hline 2 & 0.30 & 27.338 & 0.10 & 0.22 & $3.743 \times 10^{-3}$ \\
\hline 2 & 0.30 & 27.078 & 0.10 & 0.30 & $5.977 \times 10^{-3}$ \\
\hline 2 & 0.30 & 26.752 & 0.10 & 0.40 & $8.915 \times 10^{-3}$ \\
\hline 2 & 0.30 & 27.092 & 0.25 & 0.02 & $5.077 \times 10^{-4}$ \\
\hline 2 & 0.30 & 26.961 & 0.25 & 0.06 & $1.683 \times 10^{-3}$ \\
\hline 2 & 0.30 & 26.832 & 0.25 & 0.10 & $2.920 \times 10^{-3}$ \\
\hline 2 & 0.30 & 26.669 & 0.25 & 0.15 & $4.613 \times 10^{-3}$ \\
\hline 2 & 0.30 & 26.441 & 0.25 & 0.22 & $7.348 \times 10^{-3}$ \\
\hline 2 & 0.30 & 26.181 & 0.25 & 0.30 & $1.118 \times 10^{-2}$ \\
\hline 2 & 0.30 & 25.856 & 0.25 & 0.40 & $1.656 \times 10^{-2}$ \\
\hline 2 & 0.30 & 25.597 & 0.50 & 0.02 & $1.090 \times 10^{-3}$ \\
\hline 2 & 0.30 & 25.467 & 0.50 & 0.06 & $3.365 \times 10^{-3}$ \\
\hline 2 & 0.30 & 25.337 & 0.50 & 0.10 & $5.846 \times 10^{-3}$ \\
\hline 2 & 0.30 & 25.174 & 0.50 & 0.15 & $9.106 \times 10^{-3}$ \\
\hline 2 & 0.30 & 24.946 & 0.50 & 0.22 & $1.439 \times 10^{-2}$ \\
\hline 2 & 0.30 & 24.686 & 0.50 & 0.30 & $2.150 \times 10^{-2}$ \\
\hline 2 & 0.30 & 24.361 & 0.50 & 0.40 & $2.983 \times 10^{-2}$ \\
\hline
\end{tabular}


Table S-2. Continuation.

\begin{tabular}{|c|c|c|c|c|c|}
\hline $\boldsymbol{W}$ & {$[\mathbf{A O T}], \mathbf{M}$} & $Z$ & {$[\mathbf{G}], \mathbf{M}$} & {$\left[\mathrm{BuNH}_{2}\right], \mathbf{M}$} & $k_{o b s}, \mathbf{s}^{-1}$ \\
\hline 2 & 0.30 & 24.102 & 0.75 & 0.02 & $1.812 \times 10^{-3}$ \\
\hline 2 & 0.30 & 23.972 & 0.75 & 0.06 & $5.678 \times 10^{-3}$ \\
\hline 2 & 0.30 & 23.842 & 0.75 & 0.10 & $9.763 \times 10^{-3}$ \\
\hline 2 & 0.30 & 23.679 & 0.75 & 0.15 & $1.586 \times 10^{-2}$ \\
\hline 2 & 0.30 & 23.451 & 0.75 & 0.22 & $2.311 \times 10^{-2}$ \\
\hline 2 & 0.30 & 23.191 & 0.75 & 0.30 & $3.416 \times 10^{-2}$ \\
\hline 2 & 0.30 & 22.866 & 0.75 & 0.40 & $4.918 \times 10^{-2}$ \\
\hline 2 & 0.30 & 22.608 & 1.00 & 0.02 & $2.723 \times 10^{-3}$ \\
\hline 2 & 0.30 & 22.477 & 1.00 & 0.06 & $8.290 \times 10^{-3}$ \\
\hline 2 & 0.30 & 22.347 & 1.00 & 0.10 & $1.378 \times 10^{-2}$ \\
\hline 2 & 0.30 & 22.185 & 1.00 & 0.15 & $2.181 \times 10^{-2}$ \\
\hline 2 & 0.30 & 21.956 & 1.00 & 0.22 & $3.428 \times 10^{-2}$ \\
\hline 2 & 0.30 & 21.697 & 1.00 & 0.30 & $4.712 \times 10^{-2}$ \\
\hline 2 & 0.30 & 21.371 & 1.00 & 0.40 & $6.721 \times 10^{-2}$ \\
\hline 2 & 0.45 & 17.641 & 0.00 & 0.06 & $3.190 \times 10^{-4}$ \\
\hline 2 & 0.45 & 17.555 & 0.00 & 0.10 & $5.928 \times 10^{-4}$ \\
\hline 2 & 0.45 & 17.446 & 0.00 & 0.15 & $1.076 \times 10^{-3}$ \\
\hline 2 & 0.45 & 17.294 & 0.00 & 0.22 & $2.004 \times 10^{-3}$ \\
\hline 2 & 0.45 & 17.121 & 0.00 & 0.30 & $3.333 \times 10^{-3}$ \\
\hline 2 & 0.45 & 17.243 & 0.10 & 0.06 & $7.370 \times 10^{-4}$ \\
\hline 2 & 0.45 & 17.156 & 0.10 & 0.10 & $1.380 \times 10^{-3}$ \\
\hline 2 & 0.45 & 17.048 & 0.10 & 0.15 & $2.267 \times 10^{-3}$ \\
\hline 2 & 0.45 & 16.895 & 0.10 & 0.22 & $3.834 \times 10^{-3}$ \\
\hline 2 & 0.45 & 16.722 & 0.10 & 0.30 & $6.018 \times 10^{-3}$ \\
\hline
\end{tabular}


Table S-2. Continuation.

\begin{tabular}{|c|c|c|c|c|c|}
\hline $\boldsymbol{W}$ & {$[\mathrm{AOT}], \mathbf{M}$} & $Z$ & {$[\mathbf{G}], \mathbf{M}$} & {$\left[\mathrm{BuNH}_{2}\right], \mathbf{M}$} & $k_{o b s}, \mathbf{s}^{-1}$ \\
\hline 2 & 0.45 & 16.645 & 0.25 & 0.06 & $1.612 \times 10^{-3}$ \\
\hline 2 & 0.45 & 16.558 & 0.25 & 0.10 & $3.256 \times 10^{-3}$ \\
\hline 2 & 0.45 & 16.450 & 0.25 & 0.15 & $5.037 \times 10^{-3}$ \\
\hline 2 & 0.45 & 16.298 & 0.25 & 0.22 & $7.808 \times 10^{-3}$ \\
\hline 2 & 0.45 & 16.124 & 0.25 & 0.30 & $1.130 \times 10^{-2}$ \\
\hline 2 & 0.45 & 15.648 & 0.50 & 0.06 & $3.662 \times 10^{-3}$ \\
\hline 2 & 0.45 & 15.562 & 0.50 & 0.10 & $6.201 \times 10^{-3}$ \\
\hline 2 & 0.45 & 15.453 & 0.50 & 0.15 & $9.271 \times 10^{-3}$ \\
\hline 2 & 0.45 & 15.301 & 0.50 & 0.22 & $1.524 \times 10^{-2}$ \\
\hline 2 & 0.45 & 15.128 & 0.50 & 0.30 & $2.178 \times 10^{-2}$ \\
\hline 2 & 0.45 & 14.652 & 0.75 & 0.06 & $6.170 \times 10^{-3}$ \\
\hline 2 & 0.45 & 14.565 & 0.75 & 0.10 & $1.029 \times 10^{-2}$ \\
\hline 2 & 0.45 & 14.457 & 0.75 & 0.15 & $1.632 \times 10^{-2}$ \\
\hline 2 & 0.45 & 14.305 & 0.75 & 0.22 & $2.515 \times 10^{-2}$ \\
\hline 2 & 0.45 & 14.131 & 0.75 & 0.30 & $3.593 \times 10^{-2}$ \\
\hline 2 & 0.45 & 13.655 & 1.00 & 0.06 & $8.774 \times 10^{-3}$ \\
\hline 2 & 0.45 & 13.569 & 1.00 & 0.10 & $1.495 \times 10^{-2}$ \\
\hline 2 & 0.45 & 13.460 & 1.00 & 0.15 & $2.382 \times 10^{-2}$ \\
\hline 2 & 0.45 & 13.308 & 1.00 & 0.22 & $3.653 \times 10^{-2}$ \\
\hline 2 & 0.45 & 13.135 & 1.00 & 0.30 & $5.019 \times 10^{-2}$ \\
\hline
\end{tabular}


Table S-2. Continuation.

\begin{tabular}{|c|c|c|c|c|c|}
\hline$W$ & {$[\mathbf{A O T}], \mathbf{M}$} & $Z$ & {$[\mathbf{G}], \mathbf{M}$} & {$\left[\mathrm{BuNH}_{2}\right], \mathbf{M}$} & $k_{o b s}, \mathbf{s}^{-1}$ \\
\hline 2 & 0.60 & 12.299 & 0.00 & 0.02 & $1.122 \times 10^{-4}$ \\
\hline 2 & 0.60 & 12.234 & 0.00 & 0.06 & $4.012 \times 10^{-4}$ \\
\hline 2 & 0.60 & 12.169 & 0.00 & 0.10 & $7.639 \times 10^{-4}$ \\
\hline 2 & 0.60 & 12.087 & 0.00 & 0.15 & $1.322 \times 10^{-3}$ \\
\hline 2 & 0.60 & 11.973 & 0.00 & 0.22 & $2.401 \times 10^{-3}$ \\
\hline 2 & 0.60 & 11.843 & 0.00 & 0.30 & $3.848 \times 10^{-3}$ \\
\hline 2 & 0.60 & 11.681 & 0.00 & 0.40 & $6.221 \times 10^{-3}$ \\
\hline 2 & 0.60 & 12.000 & 0.10 & 0.02 & $2.506 \times 10^{-4}$ \\
\hline 2 & 0.60 & 11.935 & 0.10 & 0.06 & $8.544 \times 10^{-4}$ \\
\hline 2 & 0.60 & 11.870 & 0.10 & 0.10 & $1.517 \times 10^{-3}$ \\
\hline 2 & 0.60 & 11.788 & 0.10 & 0.15 & $2.534 \times 10^{-3}$ \\
\hline 2 & 0.60 & 11.674 & 0.10 & 0.22 & $4.166 \times 10^{-3}$ \\
\hline 2 & 0.60 & 11.544 & 0.10 & 0.30 & $6.385 \times 10^{-3}$ \\
\hline 2 & 0.60 & 11.382 & 0.10 & 0.40 & $9.918 \times 10^{-3}$ \\
\hline 2 & 0.60 & 11.551 & 0.25 & 0.02 & $5.791 \times 10^{-4}$ \\
\hline 2 & 0.60 & 11.486 & 0.25 & 0.06 & $1.755 \times 10^{-3}$ \\
\hline 2 & 0.60 & 11.421 & 0.25 & 0.10 & $3.134 \times 10^{-3}$ \\
\hline 2 & 0.60 & 11.340 & 0.25 & 0.15 & $5.006 \times 10^{-3}$ \\
\hline 2 & 0.60 & 11.226 & 0.25 & 0.22 & $7.778 \times 10^{-3}$ \\
\hline 2 & 0.60 & 11.096 & 0.25 & 0.30 & $1.185 \times 10^{-2}$ \\
\hline 2 & 0.60 & 10.933 & 0.25 & 0.40 & $1.774 \times 10^{-2}$ \\
\hline
\end{tabular}


Table S-2. Continuation.

\begin{tabular}{|c|c|c|c|c|c|}
\hline $\boldsymbol{W}$ & {$[\mathbf{A O T}], \mathbf{M}$} & $Z$ & {$[\mathbf{G}], \mathbf{M}$} & {$\left[\mathrm{BuNH}_{2}\right], \mathbf{M}$} & $k_{o b s}, s^{-1}$ \\
\hline 2 & 0.60 & 10.804 & 0.50 & 0.02 & $1.219 \times 10^{-3}$ \\
\hline 2 & 0.60 & 10.739 & 0.50 & 0.06 & $4.022 \times 10^{-3}$ \\
\hline 2 & 0.60 & 10.674 & 0.50 & 0.10 & $6.514 \times 10^{-3}$ \\
\hline 2 & 0.60 & 10.593 & 0.50 & 0.15 & $9.938 \times 10^{-3}$ \\
\hline 2 & 0.60 & 10.479 & 0.50 & 0.22 & $1.594 \times 10^{-2}$ \\
\hline 2 & 0.60 & 10.349 & 0.50 & 0.30 & $2.321 \times 10^{-2}$ \\
\hline 2 & 0.60 & 10.186 & 0.50 & 0.40 & $3.280 \times 10^{-2}$ \\
\hline 2 & 0.60 & 10.057 & 0.75 & 0.02 & $2.323 \times 10^{-3}$ \\
\hline 2 & 0.60 & 9.992 & 0.75 & 0.06 & $6.064 \times 10^{-3}$ \\
\hline 2 & 0.60 & 9.927 & 0.75 & 0.10 & $1.005 \times 10^{-2}$ \\
\hline 2 & 0.60 & 9.845 & 0.75 & 0.15 & $1.633 \times 10^{-2}$ \\
\hline 2 & 0.60 & 9.731 & 0.75 & 0.22 & $2.712 \times 10^{-2}$ \\
\hline 2 & 0.60 & 9.601 & 0.75 & 0.30 & $3.922 \times 10^{-2}$ \\
\hline 2 & 0.60 & 9.439 & 0.75 & 0.40 & $5.617 \times 10^{-2}$ \\
\hline 2 & 0.60 & 9.309 & 1.00 & 0.02 & $3.212 \times 10^{-3}$ \\
\hline 2 & 0.60 & 9.244 & 1.00 & 0.06 & $9.743 \times 10^{-3}$ \\
\hline 2 & 0.60 & 9.179 & 1.00 & 0.10 & $1.682 \times 10^{-2}$ \\
\hline 2 & 0.60 & 9.098 & 1.00 & 0.15 & $2.808 \times 10^{-2}$ \\
\hline 2 & 0.60 & 8.984 & 1.00 & 0.22 & $3.895 \times 10^{-2}$ \\
\hline 2 & 0.60 & 8.854 & 1.00 & 0.30 & $5.765 \times 10^{-2}$ \\
\hline 2 & 0.60 & 8.691 & 1.00 & 0.40 & $7.931 \times 10^{-2}$ \\
\hline
\end{tabular}


Table S-3. Influence of $n$-butylamine concentration of $k_{o b s}$ for butylaminolysis of NPC in $\mathrm{AOT} /$ chlorobenzene/water microemulsions in the presence of different glyme concentrations. $[\mathrm{NPC}]=5.0 \times 10^{-5} \mathrm{M} . \mathrm{T}=25.0^{\circ} \mathrm{C} . W=6$.

\begin{tabular}{|c|c|c|c|c|c|}
\hline $\boldsymbol{W}$ & {$[\mathrm{AOT}], \mathbf{M}$} & $Z$ & {$[\mathbf{G}], \mathbf{M}$} & {$\left[\mathrm{BuNH}_{2}\right], \mathbf{M}$} & $k_{o b s}, \mathbf{s}^{-1}$ \\
\hline 6 & 0.10 & 92.249 & 0.00 & 0.10 & $2.676 \times 10^{-4}$ \\
\hline 6 & 0.10 & 91.761 & 0.00 & 0.15 & $5.472 \times 10^{-4}$ \\
\hline 6 & 0.10 & 91.077 & 0.00 & 0.22 & $1.143 \times 10^{-3}$ \\
\hline 6 & 0.10 & 90.297 & 0.00 & 0.30 & $2.230 \times 10^{-3}$ \\
\hline 6 & 0.10 & 89.321 & 0.00 & 0.40 & $3.924 \times 10^{-3}$ \\
\hline 6 & 0.10 & 90.456 & 0.10 & 0.10 & $1.106 \times 10^{-3}$ \\
\hline 6 & 0.10 & 89.968 & 0.10 & 0.15 & $1.964 \times 10^{-3}$ \\
\hline 6 & 0.10 & 89.283 & 0.10 & 0.22 & $3.201 \times 10^{-3}$ \\
\hline 6 & 0.10 & 88.504 & 0.10 & 0.30 & $5.496 \times 10^{-3}$ \\
\hline 6 & 0.10 & 87.528 & 0.10 & 0.40 & $8.262 \times 10^{-3}$ \\
\hline 6 & 0.10 & 87.765 & 0.25 & 0.10 & $2.390 \times 10^{-3}$ \\
\hline 6 & 0.10 & 87.277 & 0.25 & 0.15 & $3.569 \times 10^{-3}$ \\
\hline 6 & 0.10 & 86.593 & 0.25 & 0.22 & $5.729 \times 10^{-3}$ \\
\hline 6 & 0.10 & 85.813 & 0.25 & 0.30 & $9.089 \times 10^{-3}$ \\
\hline 6 & 0.10 & 84.837 & 0.25 & 0.40 & $1.300 \times 10^{-2}$ \\
\hline 6 & 0.15 & 60.194 & 0.00 & 0.06 & $1.385 \times 10^{-4}$ \\
\hline 6 & 0.15 & 59.609 & 0.00 & 0.15 & $5.770 \times 10^{-4}$ \\
\hline 6 & 0.15 & 58.633 & 0.00 & 0.30 & $2.164 \times 10^{-3}$ \\
\hline 6 & 0.15 & 57.982 & 0.00 & 0.40 & $3.831 \times 10^{-3}$ \\
\hline 6 & 0.15 & 58.998 & 0.10 & 0.06 & $6.237 \times 10^{-4}$ \\
\hline
\end{tabular}


Table S-3. Continuation.

\begin{tabular}{|c|c|c|c|c|c|}
\hline $\boldsymbol{W}$ & {$[\mathrm{AOT}], \mathbf{M}$} & $Z$ & {$[\mathbf{G}], \mathbf{M}$} & {$\left[\mathrm{BuNH}_{2}\right], \mathbf{M}$} & $k_{o b s}, \mathbf{s}^{-1}$ \\
\hline 6 & 0.15 & 58.413 & 0.10 & 0.15 & $1.938 \times 10^{-3}$ \\
\hline 6 & 0.15 & 57.437 & 0.10 & 0.30 & $5.064 \times 10^{-3}$ \\
\hline 6 & 0.15 & 56.786 & 0.10 & 0.40 & $8.179 \times 10^{-3}$ \\
\hline 6 & 0.15 & 57.204 & 0.25 & 0.06 & $1.429 \times 10^{-3}$ \\
\hline 6 & 0.15 & 56.619 & 0.25 & 0.15 & $3.982 \times 10^{-3}$ \\
\hline 6 & 0.15 & 55.643 & 0.25 & 0.30 & $9.134 \times 10^{-3}$ \\
\hline 6 & 0.15 & 54.993 & 0.25 & 0.40 & $1.409 \times 10^{-2}$ \\
\hline 6 & 0.15 & 54.215 & 0.50 & 0.06 & $2.994 \times 10^{-3}$ \\
\hline 6 & 0.15 & 53.630 & 0.50 & 0.15 & $8.036 \times 10^{-3}$ \\
\hline 6 & 0.15 & 52.654 & 0.50 & 0.30 & $1.796 \times 10^{-2}$ \\
\hline 6 & 0.15 & 52.003 & 0.50 & 0.40 & $2.575 \times 10^{-2}$ \\
\hline 6 & 0.20 & 43.776 & 0.00 & 0.10 & $3.122 \times 10^{-4}$ \\
\hline 6 & 0.20 & 43.532 & 0.00 & 0.15 & $5.766 \times 10^{-4}$ \\
\hline 6 & 0.20 & 43.190 & 0.00 & 0.22 & $1.716 \times 10^{-3}$ \\
\hline 6 & 0.20 & 42.801 & 0.00 & 0.30 & $2.153 \times 10^{-3}$ \\
\hline 6 & 0.20 & 42.313 & 0.00 & 0.40 & $3.665 \times 10^{-3}$ \\
\hline 6 & 0.20 & 42.880 & 0.10 & 0.10 & $1.118 \times 10^{-3}$ \\
\hline 6 & 0.20 & 42.636 & 0.10 & 0.15 & $1.866 \times 10^{-3}$ \\
\hline 6 & 0.20 & 42.293 & 0.10 & 0.22 & $3.107 \times 10^{-3}$ \\
\hline 6 & 0.20 & 41.904 & 0.10 & 0.30 & $4.801 \times 10^{-3}$ \\
\hline 6 & 0.20 & 41.416 & 0.10 & 0.40 & $7.408 \times 10^{-3}$ \\
\hline
\end{tabular}


Table S-3. Continuation.

\begin{tabular}{|c|c|c|c|c|c|}
\hline$W$ & {$[\mathbf{A O T}], \mathbf{M}$} & $Z$ & {$[\mathbf{G}], \mathbf{M}$} & {$\left[\mathrm{BuNH}_{2}\right], \mathbf{M}$} & $k_{o b s}, \mathbf{s}^{-1}$ \\
\hline 6 & 0.20 & 41.534 & 0.25 & 0.10 & $2.462 \times 10^{-3}$ \\
\hline 6 & 0.20 & 41.290 & 0.25 & 0.15 & $3.829 \times 10^{-3}$ \\
\hline 6 & 0.20 & 40.948 & 0.25 & 0.22 & $5.963 \times 10^{-3}$ \\
\hline 6 & 0.20 & 40.558 & 0.25 & 0.30 & $8.864 \times 10^{-3}$ \\
\hline 6 & 0.20 & 40.070 & 0.25 & 0.40 & $1.337 \times 10^{-2}$ \\
\hline 6 & 0.20 & 39.292 & 0.50 & 0.10 & $5.017 \times 10^{-3}$ \\
\hline 6 & 0.20 & 39.048 & 0.50 & 0.15 & $8.180 \times 10^{-3}$ \\
\hline 6 & 0.20 & 38.706 & 0.50 & 0.22 & $1.224 \times 10^{-2}$ \\
\hline 6 & 0.20 & 38.316 & 0.50 & 0.30 & $1.787 \times 10^{-2}$ \\
\hline 6 & 0.20 & 37.828 & 0.50 & 0.40 & $2.577 \times 10^{-2}$ \\
\hline 6 & 0.20 & 37.050 & 0.75 & 0.10 & $7.874 \times 10^{-3}$ \\
\hline 6 & 0.20 & 36.806 & 0.75 & 0.15 & $1.216 \times 10^{-2}$ \\
\hline 6 & 0.20 & 36.464 & 0.75 & 0.22 & $1.911 \times 10^{-2}$ \\
\hline 6 & 0.20 & 36.074 & 0.75 & 0.30 & $2.721 \times 10^{-2}$ \\
\hline 6 & 0.20 & 35.586 & 0.75 & 0.40 & $3.758 \times 10^{-2}$ \\
\hline 6 & 0.30 & 27.879 & 0.00 & 0.02 & $5.681 \times 10^{-5}$ \\
\hline 6 & 0.30 & 27.749 & 0.00 & 0.06 & $1.905 \times 10^{-4}$ \\
\hline 6 & 0.30 & 27.619 & 0.00 & 0.10 & $3.755 \times 10^{-4}$ \\
\hline 6 & 0.30 & 27.456 & 0.00 & 0.15 & $6.661 \times 10^{-4}$ \\
\hline 6 & 0.30 & 27.228 & 0.00 & 0.22 & $1.246 \times 10^{-3}$ \\
\hline 6 & 0.30 & 26.968 & 0.00 & 0.30 & $2.168 \times 10^{-3}$ \\
\hline 6 & 0.30 & 26.643 & 0.00 & 0.40 & $3.595 \times 10^{-3}$ \\
\hline
\end{tabular}


Table S-3. Continuation.

\begin{tabular}{|c|c|c|c|c|c|}
\hline $\boldsymbol{W}$ & {$[\mathrm{AOT}], \mathbf{M}$} & $Z$ & {$[\mathbf{G}], \mathbf{M}$} & {$\left[\mathrm{BuNH}_{2}\right], \mathbf{M}$} & $k_{o b s}, \mathbf{s}^{-1}$ \\
\hline 6 & 0.30 & 27.281 & 0.10 & 0.02 & $1.747 \times 10^{-4}$ \\
\hline 6 & 0.30 & 27.151 & 0.10 & 0.06 & $6.378 \times 10^{-4}$ \\
\hline 6 & 0.30 & 27.021 & 0.10 & 0.10 & $1.063 \times 10^{-3}$ \\
\hline 6 & 0.30 & 26.858 & 0.10 & 0.15 & $1.821 \times 10^{-3}$ \\
\hline 6 & 0.30 & 26.630 & 0.10 & 0.22 & $3.037 \times 10^{-3}$ \\
\hline 6 & 0.30 & 26.370 & 0.10 & 0.30 & $4.617 \times 10^{-3}$ \\
\hline 6 & 0.30 & 26.045 & 0.10 & 0.40 & $7.022 \times 10^{-3}$ \\
\hline 6 & 0.30 & 26.384 & 0.25 & 0.02 & $5.020 \times 10^{-4}$ \\
\hline 6 & 0.30 & 26.254 & 0.25 & 0.06 & $1.515 \times 10^{-3}$ \\
\hline 6 & 0.30 & 26.124 & 0.25 & 0.10 & $2.414 \times 10^{-3}$ \\
\hline 6 & 0.30 & 25.961 & 0.25 & 0.15 & $3.722 \times 10^{-3}$ \\
\hline 6 & 0.30 & 25.733 & 0.25 & 0.22 & $5.799 \times 10^{-3}$ \\
\hline 6 & 0.30 & 25.474 & 0.25 & 0.30 & $8.381 \times 10^{-3}$ \\
\hline 6 & 0.30 & 25.148 & 0.25 & 0.40 & $1.277 \times 10^{-2}$ \\
\hline 6 & 0.30 & 24.890 & 0.50 & 0.02 & $1.031 \times 10^{-3}$ \\
\hline 6 & 0.30 & 24.759 & 0.50 & 0.06 & $2.909 \times 10^{-3}$ \\
\hline 6 & 0.30 & 24.629 & 0.50 & 0.10 & $4.871 \times 10^{-3}$ \\
\hline 6 & 0.30 & 24.467 & 0.50 & 0.15 & $7.740 \times 10^{-3}$ \\
\hline 6 & 0.30 & 24.239 & 0.50 & 0.22 & $1.154 \times 10^{-2}$ \\
\hline 6 & 0.30 & 23.979 & 0.50 & 0.30 & $1.725 \times 10^{-2}$ \\
\hline 6 & 0.30 & 23.653 & 0.50 & 0.40 & $2.479 \times 10^{-2}$ \\
\hline
\end{tabular}


Table S-3. Continuation.

\begin{tabular}{|c|c|c|c|c|c|}
\hline $\boldsymbol{W}$ & {$[\mathrm{AOT}], \mathbf{M}$} & $Z$ & {$[\mathbf{G}], \mathbf{M}$} & {$\left[\mathrm{BuNH}_{2}\right], \mathbf{M}$} & $k_{o b s}, \mathbf{s}^{-1}$ \\
\hline 6 & 0.30 & 23.395 & 0.75 & 0.02 & $1.393 \times 10^{-3}$ \\
\hline 6 & 0.30 & 23.265 & 0.75 & 0.06 & $4.390 \times 10^{-3}$ \\
\hline 6 & 0.30 & 23.135 & 0.75 & 0.10 & $8.067 \times 10^{-3}$ \\
\hline 6 & 0.30 & 22.972 & 0.75 & 0.15 & $1.194 \times 10^{-2}$ \\
\hline 6 & 0.30 & 22.744 & 0.75 & 0.22 & $1.907 \times 10^{-2}$ \\
\hline 6 & 0.30 & 22.484 & 0.75 & 0.30 & $2.746 \times 10^{-2}$ \\
\hline 6 & 0.30 & 22.159 & 0.75 & 0.40 & $3.833 \times 10^{-2}$ \\
\hline 6 & 0.30 & 21.900 & 1.00 & 0.02 & $1.938 \times 10^{-3}$ \\
\hline 6 & 0.30 & 21.770 & 1.00 & 0.06 & $6.586 \times 10^{-3}$ \\
\hline 6 & 0.30 & 21.640 & 1.00 & 0.10 & $1.139 \times 10^{-2}$ \\
\hline 6 & 0.30 & 21.477 & 1.00 & 0.15 & $1.782 \times 10^{-2}$ \\
\hline 6 & 0.30 & 21.249 & 1.00 & 0.22 & $2.533 \times 10^{-2}$ \\
\hline 6 & 0.30 & 20.989 & 1.00 & 0.30 & $3.548 \times 10^{-2}$ \\
\hline 6 & 0.30 & 20.664 & 1.00 & 0.40 & $5.034 \times 10^{-2}$ \\
\hline 6 & 0.45 & 16.847 & 0.00 & 0.10 & $4.045 \times 10^{-4}$ \\
\hline 6 & 0.45 & 16.739 & 0.00 & 0.15 & $7.127 \times 10^{-4}$ \\
\hline 6 & 0.45 & 16.587 & 0.00 & 0.22 & $1.330 \times 10^{-3}$ \\
\hline 6 & 0.45 & 16.413 & 0.00 & 0.30 & $2.218 \times 10^{-3}$ \\
\hline 6 & 0.45 & 16.197 & 0.00 & 0.40 & $3.569 \times 10^{-3}$ \\
\hline 6 & 0.45 & 16.449 & 0.10 & 0.10 & $1.133 \times 10^{-3}$ \\
\hline 6 & 0.45 & 16.340 & 0.10 & 0.15 & $1.933 \times 10^{-3}$ \\
\hline 6 & 0.45 & 16.188 & 0.10 & 0.22 & $2.930 \times 10^{-3}$ \\
\hline 6 & 0.45 & 16.015 & 0.10 & 0.30 & $4.677 \times 10^{-3}$ \\
\hline 6 & 0.45 & 15.798 & 0.10 & 0.40 & $7.085 \times 10^{-3}$ \\
\hline
\end{tabular}


Table S-3. Continuation.

\begin{tabular}{|c|c|c|c|c|c|}
\hline $\boldsymbol{W}$ & {$[\mathrm{AOT}], \mathbf{M}$} & $Z$ & {$[\mathbf{G}], \mathbf{M}$} & {$\left[\mathrm{BuNH}_{2}\right], \mathbf{M}$} & $k_{o b s}, \mathrm{~s}^{-1}$ \\
\hline 6 & 0.45 & 15.851 & 0.25 & 0.10 & $2.444 \times 10^{-3}$ \\
\hline 6 & 0.45 & 15.742 & 0.25 & 0.15 & $4.053 \times 10^{-3}$ \\
\hline 6 & 0.45 & 15.590 & 0.25 & 0.22 & $5.707 \times 10^{-3}$ \\
\hline 6 & 0.45 & 15.417 & 0.25 & 0.30 & $8.253 \times 10^{-3}$ \\
\hline 6 & 0.45 & 15.200 & 0.25 & 0.40 & $1.291 \times 10^{-2}$ \\
\hline 6 & 0.45 & 14.854 & 0.50 & 0.10 & $5.158 \times 10^{-3}$ \\
\hline 6 & 0.45 & 14.746 & 0.50 & 0.15 & $8.307 \times 10^{-3}$ \\
\hline 6 & 0.45 & 14.594 & 0.50 & 0.22 & $1.219 \times 10^{-2}$ \\
\hline 6 & 0.45 & 14.420 & 0.50 & 0.30 & $1.838 \times 10^{-2}$ \\
\hline 6 & 0.45 & 14.204 & 0.50 & 0.40 & $2.536 \times 10^{-2}$ \\
\hline 6 & 0.45 & 13.858 & 0.75 & 0.10 & $8.821 \times 10^{-3}$ \\
\hline 6 & 0.45 & 13.749 & 0.75 & 0.15 & $1.272 \times 10^{-2}$ \\
\hline 6 & 0.45 & 13.597 & 0.75 & 0.22 & $2.065 \times 10^{-2}$ \\
\hline 6 & 0.45 & 13.424 & 0.75 & 0.30 & $2.969 \times 10^{-2}$ \\
\hline 6 & 0.45 & 13.207 & 0.75 & 0.40 & $4.149 \times 10^{-2}$ \\
\hline 6 & 0.45 & 12.861 & 1.00 & 0.10 & $1.206 \times 10^{-2}$ \\
\hline 6 & 0.45 & 12.753 & 1.00 & 0.15 & $1.916 \times 10^{-2}$ \\
\hline 6 & 0.45 & 12.601 & 1.00 & 0.22 & $2.920 \times 10^{-2}$ \\
\hline 6 & 0.45 & 12.427 & 1.00 & 0.30 & $4.143 \times 10^{-2}$ \\
\hline 6 & 0.45 & 12.211 & 1.00 & 0.40 & $5.772 \times 10^{-2}$ \\
\hline
\end{tabular}


Table S-3. Continuation.

\begin{tabular}{|c|c|c|c|c|c|}
\hline $\boldsymbol{W}$ & {$[\mathrm{AOT}], \mathbf{M}$} & $Z$ & {$[\mathbf{G}], \mathbf{M}$} & {$\left[\mathrm{BuNH}_{2}\right], \mathbf{M}$} & $k_{o b s}, \mathbf{s}^{-1}$ \\
\hline 6 & 0.60 & 11.591 & 0.00 & 0.02 & $8.731 \times 10^{-5}$ \\
\hline 6 & 0.60 & 11.380 & 0.00 & 0.15 & $9.341 \times 10^{-4}$ \\
\hline 6 & 0.60 & 11.266 & 0.00 & 0.22 & $1.752 \times 10^{-3}$ \\
\hline 6 & 0.60 & 11.136 & 0.00 & 0.30 & $2.792 \times 10^{-3}$ \\
\hline 6 & 0.60 & 10.973 & 0.00 & 0.40 & $4.335 \times 10^{-3}$ \\
\hline 6 & 0.60 & 11.292 & 0.10 & 0.02 & $1.719 \times 10^{-4}$ \\
\hline 6 & 0.60 & 11.081 & 0.10 & 0.15 & $2.134 \times 10^{-3}$ \\
\hline 6 & 0.60 & 10.967 & 0.10 & 0.22 & $3.358 \times 10^{-3}$ \\
\hline 6 & 0.60 & 10.837 & 0.10 & 0.30 & $5.013 \times 10^{-3}$ \\
\hline 6 & 0.60 & 10.674 & 0.10 & 0.40 & $7.459 \times 10^{-3}$ \\
\hline 6 & 0.60 & 10.844 & 0.25 & 0.02 & $3.628 \times 10^{-4}$ \\
\hline 6 & 0.60 & 10.633 & 0.25 & 0.15 & $4.243 \times 10^{-3}$ \\
\hline 6 & 0.60 & 10.519 & 0.25 & 0.22 & $6.502 \times 10^{-3}$ \\
\hline 6 & 0.60 & 10.389 & 0.25 & 0.30 & $9.280 \times 10^{-3}$ \\
\hline 6 & 0.60 & 10.226 & 0.25 & 0.40 & $1.445 \times 10^{-2}$ \\
\hline 6 & 0.60 & 10.097 & 0.50 & 0.02 & $7.983 \times 10^{-4}$ \\
\hline 6 & 0.60 & 9.885 & 0.50 & 0.15 & $8.858 \times 10^{-3}$ \\
\hline 6 & 0.60 & 9.771 & 0.50 & 0.22 & $1.290 \times 10^{-2}$ \\
\hline 6 & 0.60 & 9.641 & 0.50 & 0.30 & $1.899 \times 10^{-2}$ \\
\hline 6 & 0.60 & 9.479 & 0.50 & 0.40 & $2.861 \times 10^{-2}$ \\
\hline
\end{tabular}


Table S-3. Continuation.

\begin{tabular}{cccccc}
\hline $\boldsymbol{W}$ & {$[\mathrm{AOT}], \mathbf{M}$} & $\boldsymbol{Z}$ & {$[\mathbf{G}], \mathbf{M}$} & {$\left[\mathrm{BuNH}_{2}\right], \mathbf{M}$} & $\boldsymbol{k}_{\text {obs }}, \mathbf{s}^{-1}$ \\
\hline 6 & 0.60 & 9.349 & 0.75 & 0.02 & $1.801 \times 10^{-3}$ \\
6 & 0.60 & 9.138 & 0.75 & 0.15 & $1.433 \times 10^{-2}$ \\
6 & 0.60 & 9.024 & 0.75 & 0.22 & $2.189 \times 10^{-2}$ \\
6 & 0.60 & 8.894 & 0.75 & 0.30 & $2.885 \times 10^{-2}$ \\
6 & 0.60 & 8.731 & 0.75 & 0.40 & $4.568 \times 10^{-2}$ \\
6 & 0.60 & 9.954 & 1.00 & 0.02 & $1.148 \times 10^{-3}$ \\
6 & 0.60 & 9.743 & 1.00 & 0.15 & $1.461 \times 10^{-2}$ \\
6 & 0.60 & 9.629 & 1.00 & 0.22 & $2.407 \times 10^{-2}$ \\
6 & 0.60 & 9.499 & 1.00 & 0.30 & $3.504 \times 10^{-2}$ \\
6 & 0.60 & 9.336 & 1.00 & 0.40 & $5.223 \times 10^{-2}$ \\
\hline
\end{tabular}




\section{Derivation of Kinetic Equations for Reaction in the Absence of Phase Transfer Catalyst}

$$
\begin{aligned}
& \text { Amine balance } \\
& {\left[\mathrm{BuNH}_{2}\right]_{T}=\left[B u N H_{2}\right]_{o}+\left[\mathrm{BuNH}_{2}\right]_{i}+\left[B u N H_{2}\right]_{w} \quad K_{o i}^{A}=\frac{\left[B u N H_{2}\right]_{i}}{\left[B u N H_{2}\right]_{o}} Z, K_{w i}^{A}=\frac{\left[B u N H_{2}\right]_{i}}{\left[B u N H_{2}\right]_{w}} W} \\
& {\left[\mathrm{BuNH}_{2}\right]_{T}=\left[B u N H_{2}\right]_{o}+\frac{K_{o i}^{A}\left[B u N H_{2}\right]_{o}}{Z}+\frac{K_{o i}^{A} W\left[B u N H_{2}\right]_{o}}{K_{w i}^{A} Z}=\left[B u N H_{2}\right]_{o}\left(1+\frac{K_{o i}^{A}}{Z}+\frac{K_{o i}^{A} W}{K_{w i}^{A} Z}\right)} \\
& {\left[B u N H_{2}\right]_{o}=\frac{\left[B u N H_{2}\right]_{T}}{1+\frac{K_{o i}^{A}}{Z}+\frac{K_{o i}^{A} W}{K_{w i}^{A} Z}} \quad K_{o w}^{A}=\frac{K_{o i}^{A}}{K_{w i}^{A}} \quad\left[B u N H_{2}\right]_{o}=\frac{Z\left[B u N H_{2}\right]_{T}}{K_{o i}^{A}+Z+K_{o w}^{A} W}}
\end{aligned}
$$

$$
\begin{aligned}
& \text { Substrate balance } \\
& {[N P C]_{T}=[N P C]_{o}+[N P C]_{i}+\left[T^{ \pm}\right]_{o}}
\end{aligned}
$$

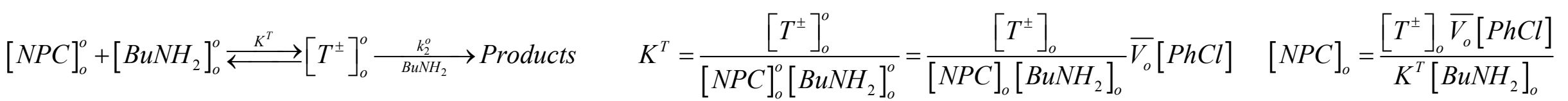

$$
\begin{aligned}
& K_{o i}^{N P C}=\frac{[N P C]_{i}}{[N P C]_{o}} Z \quad[N P C]_{i}=\frac{K_{o i}^{N P C}}{Z}[N P C]_{o}=\frac{K_{o i}^{N P C}\left[T^{ \pm}\right]_{o} \overline{V_{o}}[P h C l]}{Z K^{T}\left[B u N H_{2}\right]_{o}}
\end{aligned}
$$


$\left[\mathrm{BuNH}_{2}\right]_{o} ;\left[\mathrm{BuNH}_{2}\right]_{i}$ and $\left[\mathrm{BuNH}_{2}\right]_{w}$ are concentrations in the continuous medium (oil), interface and water microdroplet respectively referred to the total volume of the microemulsion.

$\left[\mathrm{BuNH}_{2}\right]_{o}^{o} ;\left[\mathrm{BuNH}_{2}\right]_{\mathrm{i}}^{\mathrm{i}}$ and $\left[\mathrm{BuNH}_{2}\right]_{w}^{w}$ are concentrations in the continuous medium, interface and water microdroplet respectively referred to the volume of the phase.

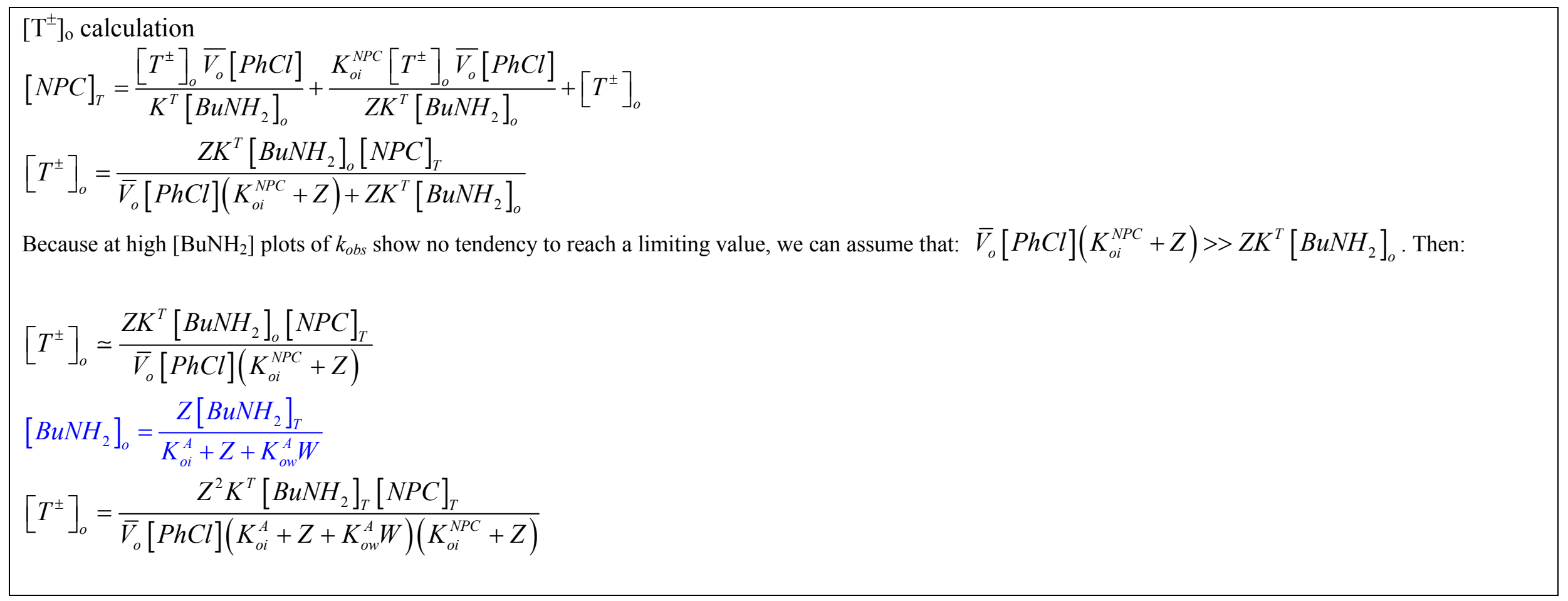




Overall rate equation
$r=k_{2}^{i}\left[B u N H_{2}\right]_{i}^{i}[N P C]_{i}+k_{2}^{o}\left[B u N H_{2}\right]_{o}^{o}\left[T^{ \pm}\right]_{o}$
$r=k_{2}^{i} \frac{\left[B u N H_{2}\right]_{i}}{\overline{V_{A O T}}[A O T]}[N P C]_{i}+k_{2}^{o} \frac{\left[B u N H_{2}\right]_{o}}{\overline{V_{o}}[P h C l]}\left[T^{ \pm}\right]_{o}$
$\left[B u N H_{2}\right]_{o}=\frac{Z\left[B u N H_{2}\right]_{T}}{K_{o i}^{A}+Z+K_{o w}^{A} W} \quad \quad K_{o i}^{A}=\frac{\left[B u N H_{2}\right]_{i}}{\left[B u N H_{2}\right]_{o}}$
$\left[T^{ \pm}\right]_{o}=\frac{Z^{2} K^{T}\left[B u N H_{2}\right]_{T}[N P C]_{T}}{\bar{V}_{o}[P h C l]\left(K_{o i}^{N P C}+Z\right)\left(K_{o i}^{A}+Z+K_{o w}^{A} W\right)}$

Substituting $\left[\mathrm{BuNH}_{2}\right]_{i} ;\left[\mathrm{BuNH}_{2}\right]_{o} ;[\mathrm{NPC}]_{i}$ and $\left[\mathrm{T}^{ \pm}\right]_{o}$ in the overall rate equation:

$k_{o b s}=\frac{k_{2}^{i} K_{o i}^{N P C} K_{o i}^{A}\left[B u N H_{2}\right]_{T}}{\bar{V}_{A O T}[A O T]\left(K_{o i}^{N P C}+Z\right)\left(K_{o i}^{A}+Z+K_{o w}^{A} W\right)}+\frac{k_{2}^{o} K^{T} Z^{3}\left[B u N H_{2}\right]_{T}^{2}}{\bar{V}_{o}^{2}[P h C l]^{2}\left(K_{o i}^{N P C}+Z\right)\left(K_{o i}^{A}+Z+K_{o w}^{A} W\right)^{2}}=k_{A}\left[B u N H_{2}\right]_{T}+k_{B}\left[B u N H_{2}\right]_{T}^{2}$ 


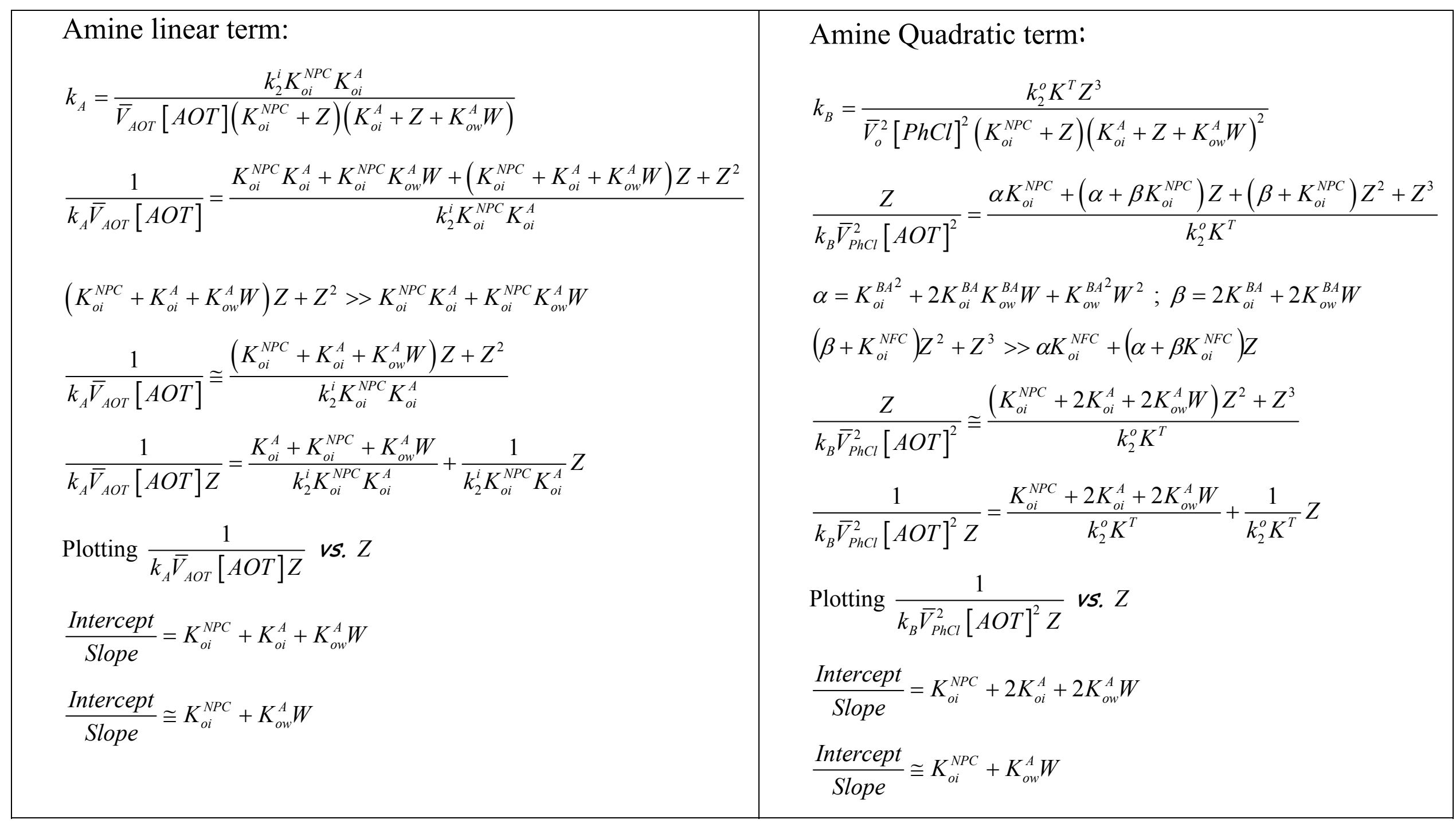




\begin{tabular}{|l|l|}
\hline Amine linear term (II): & Amine quadratic term (II): \\
Intercept $=\frac{K_{o i}^{A}+K_{o i}^{N P C}+K_{o w}^{A} W}{k_{2}^{i} K_{o i}^{N P C} K_{o i}^{A}}$ & Intercept $=\frac{K_{o i}^{N P C}+2 K_{o i}^{A}+2 K_{o w}^{A} W}{k_{2}^{o} K^{T}}$ \\
Intercept $=\frac{K_{o i}^{N P C}+K_{o i}^{A}}{k_{2}^{i} K_{o i}^{N P C} K_{o i}^{A}}+\frac{K_{o w}^{A} W}{k_{2}^{i} K_{o i}^{N P C} K_{o i}^{A}}$ & Intercept $=\frac{K_{o i}^{N P C}+2 K_{o i}^{A}}{k_{2}^{o} K^{T}+\frac{2 K_{o w}^{A} W}{k_{2}^{o} K^{T}}}$ \\
Intercept $\cong \frac{1}{k_{2}^{i} K_{o i}^{A}}+\frac{K_{o w}^{A}}{k_{2}^{i} K_{o i}^{N P C} K_{o i}^{A} W}$ & Intercept $\cong \frac{K_{o i}^{N P C}}{k_{2}^{o} K^{T}}+\frac{2 K_{o w}^{A} W}{k_{2}^{o} K^{T}}$ \\
Plotting Intercept vs. W: & Plotting Intercept vs. W: \\
Intercept' $=1 /\left(k_{2}^{i} K_{o i}^{A}\right)$ & Intercept " $\cong \frac{K_{o i}^{N P C}}{k_{2}^{o} K^{T}}$ \\
Slope' $=K_{o w}^{A} /\left(k_{2}^{i} K_{o i}^{N P C} K_{o i}^{A}\right)$ & Slope" $\cong \frac{2 K_{o w}^{A} W}{k_{2}^{o} K^{T}}$ \\
\hline Slope $=\frac{1}{k_{2}^{i} K_{o i}^{N P C} K_{o i}^{A}}$ & Slope $=\frac{1}{k_{2}^{o} K^{T}}$ \\
& \\
\hline
\end{tabular}




\section{Derivation of Kinetic Equations for Reaction in the Presence of Phase Transfer Catalyst}

Amine balance
$\left[B u N H_{2}\right]_{T}=\left[B u N H_{2}\right]_{o}+\left[B u N H_{2}\right]_{i}+\left[B u N H_{2}\right]_{w} \quad K_{o i}^{A}=\frac{\left[B u N H_{2}\right]_{i}}{\left[B u N H_{2}\right]_{o}} Z, K_{w i}^{A}=\frac{\left[B u N H_{2}\right]_{i}}{\left[B u N H_{2}\right]_{w}}$
$\left[B u N H_{2}\right]_{T}=\left[B u N H_{2}\right]_{o}+\frac{K_{o i}^{A}\left[B u N H_{2}\right]_{o}}{Z}+\frac{K_{o i}^{A} W\left[B u N H_{2}\right]_{o}}{K_{w i}^{A} Z}=\left[B u N H_{2}\right]_{o}\left(1+\frac{K_{o i}^{A}}{Z}+\frac{K_{o i}^{A} W}{K_{w i}^{A} Z}\right)$
$\left[B u N H_{2}\right]_{o}=\frac{\left[B u N H_{2}\right]_{T}}{1+\frac{K_{o i}^{A}+\frac{K_{o i}^{A} W}{Z}}{K_{w i}^{A} Z}} \quad K_{o w}^{A}=\frac{K_{o i}^{A}}{K_{w i}^{A}} \quad\left[B u N H_{2}\right]_{o}=\frac{Z\left[B u N H_{2}\right]_{T}}{K_{o i}^{A}+Z+K_{o w}^{A} W}$

Glyme balance
$[G]_{T}=[G]_{o}+[G]_{i}+[G]_{w} \quad K_{o i}^{G}=\frac{[G]_{i}}{[G]_{o}} Z, K_{w i}^{G}=\frac{[G]_{i}}{[G]_{w}} W$
$[G]_{T}=[G]_{o}+\frac{K_{o i}^{G}[G]_{o}}{Z}+\frac{K_{o i}^{G} W[G]_{o}}{K_{w i}^{G} Z}=[G]_{o}\left(1+\frac{K_{o i}^{G}}{Z}+\frac{K_{o i}^{G} W}{K_{w i}^{G} Z}\right)$
$[G]_{o}=\frac{[G]_{T}}{1+\frac{K_{o i}^{G}}{Z}+\frac{K_{o i}^{G} W}{K_{w i}^{G} Z}} \quad K_{o w}^{G}=\frac{K_{o i}^{G}}{K_{w i}^{G}}$




$$
\begin{aligned}
& \text { Substrate balance } \\
& {[N P C]_{T}=[N P C]_{o}+[N P C]_{i}+\left[T^{ \pm}\right]_{o}+\left[T^{ \pm} G\right]_{o}} \\
& \left.[\mathrm{NPC}]_{o}^{o}+[\mathrm{BuNH}]_{2}\right]_{o}^{\circ} \stackrel{K^{T}}{\longleftrightarrow}\left[T^{ \pm}\right]_{o}^{o} \frac{k_{2}^{o}}{{B u N H_{2}}_{2}} \text { Products } \quad K^{T}=\frac{\left[T^{ \pm}\right]_{o}^{o}}{[N P C]_{o}^{o}\left[\mathrm{BuNH}_{2}\right]_{o}^{o}}=\frac{\left[T^{ \pm}\right]_{o}}{[N P C]_{o}\left[\mathrm{BuNH}_{2}\right]_{o}} \overline{V_{o}}[\mathrm{PhCl}] \quad[\mathrm{NPC}]_{o}=\frac{\left[T^{ \pm}\right]_{o} \bar{V}_{o}[\mathrm{PhCl}]}{K^{T}\left[\mathrm{BuNH}_{2}\right]_{o}} \\
& K_{o i}^{N P C}=\frac{[N P C]_{i}}{[N P C]_{o}} Z \quad[N P C]_{i}=\frac{K_{o i}^{N P C}}{Z}[N P C]_{o}=\frac{K_{o i}^{N P C}\left[T^{ \pm}\right]_{o} \overline{V_{o}}[P h C l]}{Z K^{T}\left[B_{N} H_{2}\right]_{o}} \\
& {\left[T^{ \pm}\right]_{o}^{o}+[G]_{o}^{o} \stackrel{K^{T G}}{\longleftrightarrow}\left[T^{ \pm} G\right]_{o}^{o} \quad K^{T G}=\frac{\left[T^{ \pm} G\right]_{o}^{o}}{\left[T^{ \pm}\right]_{o}^{o}[G]_{o}^{o}}=\frac{\left[T^{ \pm} G\right]_{o}}{\left[T^{ \pm}\right]_{o}[G]_{o}} \bar{V}_{o}[P h C l] \quad\left[T^{ \pm} G\right]_{o}=\frac{K^{T G}\left[T^{ \pm}\right]_{o}[G]_{o}}{\overline{V_{o}}[P h C l]}}
\end{aligned}
$$




$\left[\begin{array}{l}{\left[\mathrm{T}^{ \pm}\right]_{o} \text { calculation }} \\ {[N P C]_{T}=[N P C]_{o}+[N P C]_{i}+\left[T^{ \pm}\right]_{o}+\left[T^{ \pm} G\right]_{o}} \\ {[N P C]_{T}=\frac{\left[T^{ \pm}\right]_{o}}{K^{T}\left[B u N H_{2}\right]_{o}}+\frac{\bar{V}_{o}[P h C l]}{Z K^{T}\left[B u N H_{2}\right]_{o}}+\left[T^{ \pm}\right]_{o}+\frac{K^{T G}\left[T^{ \pm}\right]_{o}[G]_{o}}{\overline{V_{o}}[P h C l]}} \\ {[N P C]_{T}=\left[T^{ \pm}\right]_{o}\left(\frac{\overline{V_{o}}[P h C l]}{K^{T}\left[B u N H_{2}\right]_{o}}+\frac{K_{o i}^{N P C} \overline{V_{o}}[P h C l]}{Z K^{T}\left[B u N H_{2}\right]_{o}}+1+\frac{K^{T G}[G]_{o}}{\overline{V_{o}}[P h C l]}\right)} \\ {[N P C]_{T}=\left[T^{ \pm}\right]_{o}\left(\frac{\bar{V}_{o}^{2}[P h C l]^{2} Z+\bar{V}_{o}^{2}[P h C l]^{2} K_{o i}^{N P C}+\overline{V_{o}}[P h C l] Z K^{T}\left[B u N H_{2}\right]_{o}}{\overline{V_{o}}[P h C l] Z K^{T}\left[B u N H_{2}\right]_{o}}+\frac{K^{T G}[G]_{o}}{\overline{V_{o}}[P h C l]}\right)} \\ {[N P C]_{T}=\left[T^{ \pm}\right]_{o}\left(\frac{\overline{V_{o}}[P h C l]\left(K_{o i}^{N P C}+Z\right)+Z K^{T}\left[B u N H_{2}\right]_{o}}{Z K^{T}\left[B u N H_{2}\right]_{o}}+\frac{K^{T G}[G]_{o}}{\overline{V_{o}}[P h C l]}\right)} \\ \text { For the reaction in the absence of phase transfer catalyst we have shown that } \bar{V}_{o}[P h C l]\left(K_{o i}^{N P C}+Z\right)>>Z K^{T}\left[B u N H_{2}\right]_{o} \\ {[N P C]_{T} \cong\left[T^{ \pm}\right]_{o}\left(\frac{\overline{V_{o}}[P h C l]\left(K_{o i}^{N P C}+Z\right)}{Z K^{T}\left[B u N H_{2}\right]_{o}}+\frac{K^{T G}[G]_{o}}{\bar{V}_{o}[P h C l]}\right)} \\ {[N P C]_{T}=\left[T^{ \pm}\right]_{o}\left(\frac{\bar{V}_{o}^{2}[P h C l]^{2}\left(K_{o i}^{N P C}+Z\right)+Z K^{T} K^{T G}\left[B u N H_{2}\right]_{o}[G]_{o}}{\overline{V_{o}}[P h C l] Z K^{T}\left[B u N H_{2}\right]_{o}}\right)}\end{array}\right.$




$$
\left[T^{ \pm}\right]_{o}=\frac{\overline{V_{o}}[\mathrm{PhCl}] \mathrm{ZK}^{T}\left[\mathrm{BuNH}_{2}\right]_{o}[\mathrm{NPC}]_{T}}{\bar{V}_{o}^{2}[\mathrm{PhCl}]^{2}\left(K_{o i}^{N P C}+Z\right)+Z K^{T} K^{T G}\left[\mathrm{BuNH}_{2}\right]_{o}[G]_{o}}
$$

Because at high $\left[\mathrm{BuNH}_{2}\right]$ or $[\mathrm{Glyme}]$ plots of $k_{o b s}$ vs. $\left[\mathrm{BuNH}_{2}\right]$ or $[\mathrm{Glyme}]$ show no tendency to reach a limiting value, we can assume that: $\bar{V}_{o}^{2}[P h C l]^{2}\left(K_{o i}^{N P C}+Z\right)>>Z K^{T} K^{T G}\left[B u N H_{2}\right]_{o}[G]_{o}$. Then:

$$
\begin{aligned}
& {\left[T^{ \pm}\right]_{o} \cong \frac{Z K^{T}\left[\mathrm{BuNH}_{2}\right]_{o}[\mathrm{NPC}]_{T}}{\overline{V_{o}}[\mathrm{PhCl}]\left(K_{o i}^{N P C}+Z\right)}} \\
& {\left[\mathrm{BuNH}_{2}\right]_{o}=\frac{Z\left[B u N H_{2}\right]_{T}}{K_{o i}^{A}+Z+K_{o w}^{A} W}}
\end{aligned}
$$$$
\left[T^{ \pm}\right]_{o}=\frac{Z^{2} K^{T}\left[B u N H_{2}\right]_{T}[N P C]_{T}}{\overline{V_{o}}[P h C l]\left(K_{o i}^{A}+Z+K_{o w}^{A} W\right)\left(K_{o i}^{N P C}+Z\right)}
$$

$$
\begin{aligned}
& \text { Overall rate equation } \\
& r=k_{2}^{i}\left[B u N H_{2}\right]_{i}^{i}[N P C]_{i}+k_{2}^{o}\left[B u N H_{2}\right]_{o}^{o}\left[T^{ \pm}\right]_{o}+\left(k_{1}^{G}\right)^{o}\left[T^{ \pm} G\right]_{o}+\left(k_{2}^{G}\right)^{o}\left[B u N H_{2}\right]_{o}^{o}\left[T^{ \pm} G\right]_{o} \\
& r=k_{2}^{i} \frac{\left[B u N H_{2}\right]_{i}}{\overline{V_{A O T}}[A O T]}[N P C]_{i}+k_{2}^{o} \frac{\left[B u N H_{2}\right]_{o}}{\overline{V_{o}}[P h C l]}\left[T^{ \pm}\right]_{o}+\left(k_{1}^{G}\right)^{o}\left[T^{ \pm} G\right]_{o}+\left(k_{2}^{G}\right)^{o} \frac{\left[B u N H_{2}\right]_{o}}{\overline{V_{o}}[P h C l]}\left[T^{ \pm} G\right]_{o}
\end{aligned}
$$




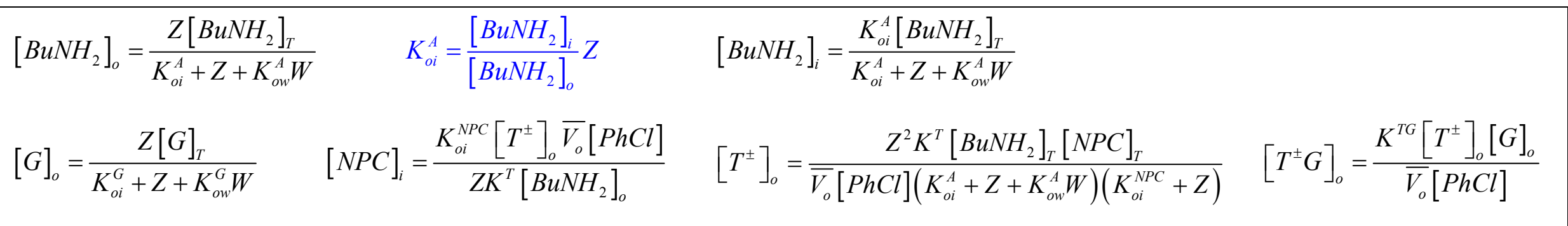

Substituting $\left.[\mathrm{BuNH}]_{2}\right]_{i} ;\left[\mathrm{BuNH}_{2}\right]_{o} ;[N P C]_{i} ;\left[T^{ \pm}\right]_{o} ;\left[T^{ \pm} G\right]_{o}$ and $[G]_{o}$ in the overall rate equation:

$$
\begin{aligned}
& k_{o b s}=\frac{k_{2}^{i} K_{o i}^{N P C} K_{o i}^{A}\left[B u N H_{2}\right]_{T}}{\bar{V}_{A O T}[A O T]\left(K_{o i}^{N P C}+Z\right)\left(K_{o i}^{A}+Z+K_{o w}^{A} W\right)}+\frac{k_{2}^{o} K^{T} Z^{3}\left[B u N H_{2}\right]_{T}^{2}}{\bar{V}_{o}^{2}[P h C l]^{2}\left(K_{o i}^{N P C}+Z\right)\left(K_{o i}^{A}+Z+K_{o w}^{A} W\right)^{2}}+ \\
& +\frac{\left(k_{1}^{G}\right)^{o} K^{T} K^{T G} Z^{3}\left[B u N H_{2}\right]_{T}[G]_{T}}{\bar{V}_{o}^{2}[P h C l]^{2}\left(K_{o i}^{N P C}+Z\right)\left(K_{o i}^{A}+Z+K_{o w}^{A} W\right)\left(K_{o i}^{G}+Z+K_{o w}^{G} W\right)}+\frac{\left(k_{2}^{G}\right)^{o} K^{T} K^{T G} Z^{4}\left[B u N H_{2}\right]_{T}^{2}[G]_{T}}{\bar{V}_{o}^{3}[P h C l]^{3}\left(K_{o i}^{N P C}+Z\right)\left(K_{o i}^{A}+Z+K_{o w}^{A} W\right)^{2}\left(K_{o i}^{G}+Z+K_{o w}^{G} W\right)} \\
& k_{o b s}=\frac{k_{2}^{i} K_{o i}^{N P C} K_{o i}^{A}\left[B u N H_{2}\right]_{T}}{\bar{V}_{A O T}[A O T]\left(K_{o i}^{N P C}+Z\right)\left(K_{o i}^{A}+Z+K_{o w}^{A} W\right)}+\frac{k_{2}^{o} K^{T} Z\left[B u N H_{2}\right]_{T}^{2}}{\bar{V}_{o}^{2}[A O T]^{2}\left(K_{o i}^{N P C}+Z\right)\left(K_{o i}^{A}+Z+K_{o w}^{A} W\right)^{2}}+ \\
& +\frac{\left(k_{1}^{G}\right)^{o} K^{T} K^{T G} Z\left[B u N H_{2}\right]_{T}[G]_{T}}{\bar{V}_{o}^{2}[A O T]^{2}\left(K_{o i}^{N P C}+Z\right)\left(K_{o i}^{A}+Z+K_{o w}^{A} W\right)\left(K_{o i}^{G}+Z+K_{o w}^{G} W\right)}+\frac{\left(k_{2}^{G}\right)^{o} K^{T} K^{T G} Z\left[B u N H_{2}\right]_{T}^{2}[G]_{T}}{\bar{V}_{o}^{3}[A O T]^{3}\left(K_{o i}^{N P C}+Z\right)\left(K_{o i}^{A}+Z+K_{o w}^{A} W\right)^{2}\left(K_{o i}^{G}+Z+K_{o w}^{G} W\right)}
\end{aligned}
$$


Plotting $k_{o b s} v s .[\mathrm{G}]_{\mathrm{T}}$ :

Slope $=\frac{\left(k_{1}^{G}\right)^{o} K^{T} K^{T G} Z\left[B u N H_{2}\right]_{T}}{\bar{V}_{o}^{2}[A O T]^{2}\left(K_{o i}^{N P C}+Z\right)\left(K_{o i}^{A}+Z+K_{o w}^{A} W\right)\left(K_{o i}^{G}+Z+K_{o w}^{G} W\right)}+\frac{\left(k_{2}^{G}\right)^{o} K^{T} K^{T G} Z\left[B u N H_{2}\right]_{T}^{2}}{\bar{V}_{o}^{3}[A O T]^{3}\left(K_{o i}^{N P C}+Z\right)\left(K_{o i}^{A}+Z+K_{o w}^{A} W\right)^{2}\left(K_{o i}^{G}+Z+K_{o w}^{G} W\right)}$

$\frac{\text { Slope }}{\left[B u N H_{2}\right]}=\frac{\left(k_{1}^{G}\right)^{o} K^{T} K^{T G} Z}{\bar{V}_{o}^{2}[A O T]^{2}\left(K_{o i}^{N P C}+Z\right)\left(K_{o i}^{A}+Z+K_{o w}^{A} W\right)\left(K_{o i}^{G}+Z+K_{o w}^{G} W\right)}+\frac{\left(k_{2}^{G}\right)^{o} K^{T} K^{T G} Z\left[B u N H_{2}\right]_{T}}{\bar{V}_{o}^{3}[A O T]^{3}\left(K_{o i}^{N P C}+Z\right)\left(K_{o i}^{A}+Z+K_{o w}^{A} W\right)^{2}\left(K_{o i}^{G}+Z+K_{o w}^{G} W\right)}$

Plotting Slope/[BuNH$\left.H_{2}\right]$ vs. $\left[\mathrm{BuNH}_{2}\right]$

Intercept $^{\prime}=k_{C}=\frac{\left(k_{1}^{G}\right)^{o} K^{T} K^{T G} Z}{\bar{V}_{o}^{2}[A O T]^{2}\left(K_{o i}^{N P C}+Z\right)\left(K_{o i}^{A}+Z+K_{o w}^{A} W\right)\left(K_{o i}^{G}+Z+K_{o w}^{G} W\right)}$
Slope $^{\prime}=k_{D}=\frac{\left(k_{2}^{G}\right)^{o} K^{T} K^{T G} Z}{\bar{V}_{o}^{3}[A O T]^{3}\left(K_{o i}^{N P C}+Z\right)\left(K_{o i}^{A}+Z+K_{o w}^{A} W\right)^{2}\left(K_{o i}^{G}+Z+K_{o w}^{G} W\right)}$ 
By means of the Intercept'/Slope' ratio $\left(k_{C} / k_{D}\right)$ and applying $\mathrm{Z}=[\mathrm{PhCl}] /[\mathrm{AOT}]$ we obtain:

$$
\frac{k_{C} / k_{D}}{\bar{V}_{o}[A O T]}=\frac{\left(k_{G}^{1}\right)_{o}\left(K_{o i}^{A}+K_{o w}^{A} W\right)}{\left(k_{G}^{2}\right)_{o}}+\frac{\left(k_{G}^{1}\right)_{o}}{\left(k_{G}^{2}\right)_{o}} Z
$$

